\title{
Anatomía Quirúrgica y Radiológica del Hígado. Fundamentos para las Resecciones Hepáticas
}

\author{
Radiological and Surgical Anatomy of the Liver and \\ Fundamentals of the Various Options Liver Resections
}

Carlos Manterola ${ }^{1,2}$; Mariano del Sol ${ }^{1}$; Nicolás Ottone ${ }^{2}$ \& Tamara Otzen ${ }^{3}$

MANTEROLA, C.; DEL SOL, M.; OTTONE, N. \& OTZEN, T. Anatomía quirúrgica y radiológica del hígado. Fundamentos para las resecciones hepáticas. Int. J. Morphol., 35(4):1525-1539, 2017.

RESUMEN: El hígado es un órgano sólido, de gran relevancia para la fisiología. Es asiento potencial de lesiones tumorales quísticas y sólidas; benignas y malignas (primarias y secundarias); razón por la cual, conocer su anatomía radiológica y quirúrgica es muy relevante. Los antecedentes históricos comienzan con Berta en 1716, quien fue el primero en realizar una resección hepática; en 1888, Lagenbuch fue el primero el realizar una resección hepática programada. En 1889, Keen realizó la primera lobectomía hepática izquierda, seguido de Webde, en 1910, quien ejecutó la primera lobectomía hepática derecha. Más tarde, Couinaud, en 1957, realizó ua descripción completa de la anatomía segmentaria del hígado, dando una mejor comprensión quirúrgica de la morfología hepática, para su abordaje en distintas patologías. Un hito fundamental en el desarrollo del estudio del hígado, fue el establecimiento de la "Clasificación de Brisbane", por parte del Comité Científico de la Asociación Internacional Hepatobilio-Pancreática, poniendo fin a la confusión terminológica establecida entre los términos franceses y anglosajones. Y desde el ámbito anatómico, se destaca la aparición de Terminologia Anatomica, por parte del Programa Federativo Internacional de Terminologia Anatomica (FIPAT) dependiente de la Federación Internacional de Asociaciones de Anatomistas (IFAA), quienes dentro de la misma, establecieron los términos anatómicos correspondientes al hígado. El objetivo de este manuscrito, es entregar un resumen esquemático de la anatomía quirúrgica y radiológica del hígado, que fundamentan las diferentes opciones de resecciones hepáticas.

PALABRAS CLAVE: Anatomía hepática; Cirugía hepática; Resecciones hepáticas; Hepatectomías; Seccionectomías.

\section{GENERALIDADES}

El hígado es un órgano multifuncional, de fisiología compleja; destacando sus funciones vasculares (de almacenamiento de hasta el $10 \%$ del volumen circulante de sangre y de filtración sinusoidal mediante el rol de las Macrophagocytus stellatus o células de Kupffer); metabólicas, propias del rol de los hepatocitos en el metabolismo de proteínas, grasas e hidratos de carbono y otros; y secretoras y excretoras (encargadas de la formación de bilis); asiento potencial de lesiones tumorales quísticas y sólidas; benignas y malignas (primarias y secundarias); razón por la cual, conocer su anatomía radiológica y quirúrgica es muy relevante (Juza \& Pauli, 2014) (Fig. 1).

Es una víscera maciza, cuyo peso oscila entre 1,4 a $1,8 \mathrm{~kg}$ en el hombre y 1,2 a $1,4 \mathrm{~kg}$ en la mujer. Se encuentra cubierto por las costillas, excepto a nivel del epigastrio (de hecho, en inspiración profunda, puede llegar al $4^{\circ}$ espacio intercostal). Se encuentra sujeto a la pared abdominal por medio de elementos de fijación (ligamentos redondo [que contiene la vena umbilical], suspensorio, coronales y triangulares derecho e izquierdo; ligamento hepatogástrico y hepatoduodenal; a los que se ha de agregar la vena cava y el pedículo hepático, compuesto por la vena porta, la arteria hepática propia y el conducto colédoco) (Bismuth, 2013; Juza \& Pauli). Su irrigación depende en un $75 \%$ de la vena porta y en el $25 \%$ restante, de la arteria hepática; sin embargo, existen variantes frecuentes: Michels I (55\%), caracterizado por una arteria hepática que nace del tronco celíaco dando dos arterias, gastroduodenal y hepática propia, que posteriormente se divide en derecha e izquierda; Michels II $(10 \%)$, caracterizado por una arteria hepática izquierda que nace de la arteria gástrica izquierda; Michels III (11\%), ca-

\footnotetext{
${ }^{1}$ Centro de Excelencia en Estudios Morfológicos y Quirúrgicos (CEMyQ), Universidad de La Frontera, Temuco, Chile.

${ }^{2}$ Programa de Doctorado en Ciencias Morfológicas, Universidad de La Frontera, Temuco, Chile.

${ }^{3}$ Facultad de Ciencias de la Salud, Universidad de Tarapacá, Arica, Chile
} 


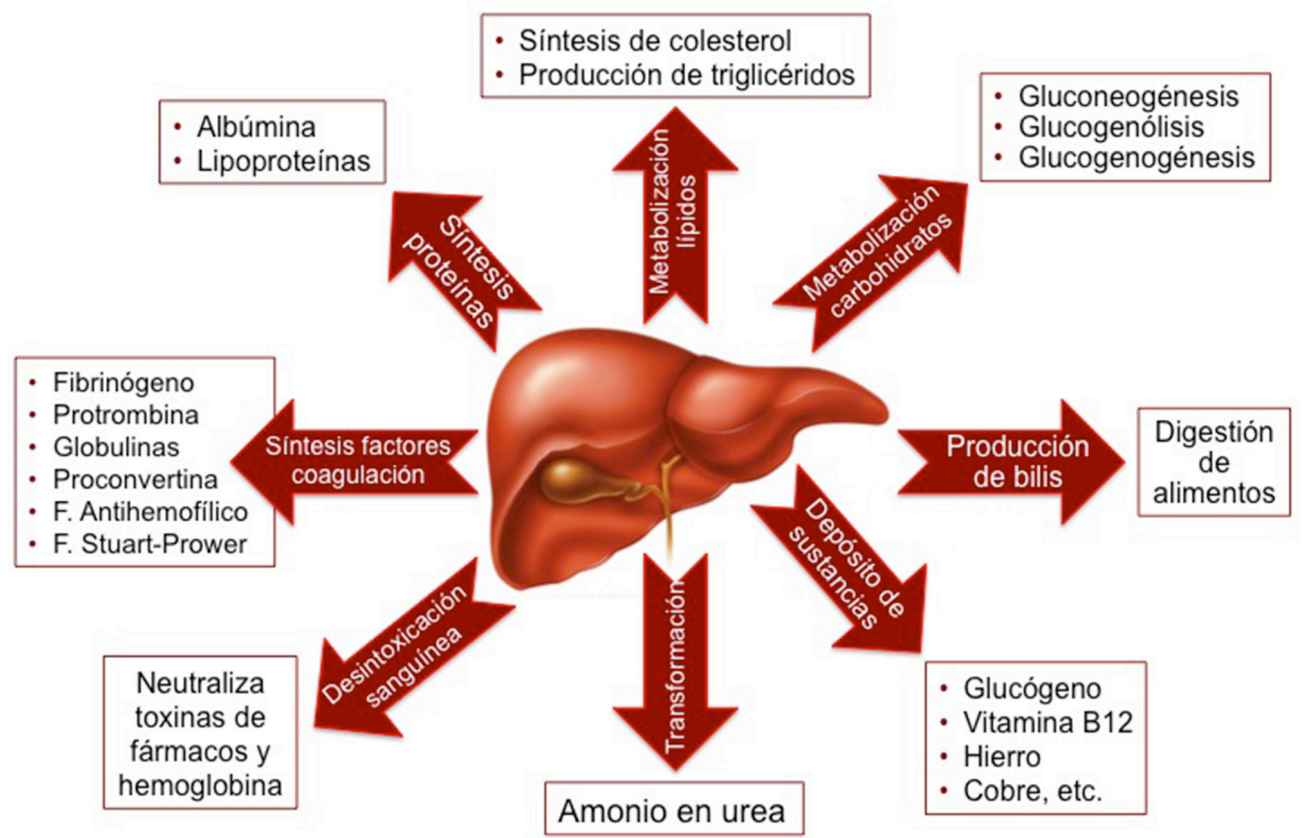

Fig. 1. Esquema de la fisiología del hígado.

racterizado por una arteria hepática derecha que se origina en la arteria mesentérica superior; Michels IV (11\%), la arteria hepática izquierda nace de la arteria gástrica izquierda y la arteria hepática derecha se origina en la arteria mesentérica superior; Michels V (8\%), en que la arteria hepática común da sus ramas (derecha e izquierda), existiendo otra accesoria para el lóbulo izquierdo, que se origina en la arteria gástrica izquierda; Michels VI (7\%), la arteria hepática común da sus ramas derecha e izquierda, existiendo otra accesoria para el lóbulo derecho, que se origina en la arteria mesentérica superior; Michels VII (1\%), que combina los tipos V y VI; Michels VIII (4\%), el lóbulo derecho es irrigado por una arteria proveniente de la mesentérica superior, y el lóbulo izquierdo por dos ramas: una proveniente de la arteria hepática común y otra de la arteria gástrica izquierda; Michels IX $(4,5 \%)$, en que la arteria hepática común se origina en la arteria mesentérica superior; Michels X $(0,5 \%)$, en que la arteria hepática común se origina en la arteria gástrica izquierda (Abdel-Misih \& Bloomston, 2010; Del Cura et al., 2010).

Desde el punto de vista de sus relaciones peritoneales, el hígado se encuentra en el compartimiento supramesocólico, pero ocupa también parte del inframesocólico (Skandalakis et al., 2004). Se divide en dos lóbulos (derecho e izquierdo), cuyo límite depende del punto de vista con que se analice: morfológico o funcional. El primero, considera al ligamento falciforme como límite entre ambos; en cambio el segundo, considera a la fisura portal principal ("línea de Cantlie") (plano medio que pasa a través del lecho de la vesícula biliar en la parte inferior, y la vena cava inferior en su parte superior). Existe además el lóbulo cuadrado (no reconocido como lóbulo verdadero por Schwartz). Este, se puede ver desde la cara inferior del hígado. Se ubica entre la fosa vesicular a la derecha, el surco umbilical a la izquierda y el hilio del hígado por detrás. Correspondiente a una porción del segmento medial izquierdo o segmento IV. Por otro lado, también se describe el lóbulo caudado (lóbulo de Spiegel), ubicado entre el margen posterior del hilio del hígado por delante y la vena porta hepática por detrás.

Es así como, continuando con el enfoque funcional, el hígado, se puede dividir en 4 secciones por las cisuras umbilical, portal principal y portal derecha. De este modo, se reconocen las secciones posterior, anterior, medial y lateral (Skandalakis et al.; Juza \& Pauli) (Fig. 2).

\section{ASPECTOS HISTÓRICOS}

Existe cierta evidencia que fue Berta quien realizó la primera resección hepática $(\mathrm{RH})$ en 1716 , oportunidad en la que habría extirpado un fragmento de hígado para tratar un desgarro hepático. Sin embargo, fue Lagenbuch en 1888 quien informó la primera resección hepática programada (una lobectomía izquierda parcial). Pero, fue Keen en 1889, quien realizó la primera lobectomía hepática izquierda; y Wendel en 1910, quien realizó la primera lobectomía hepática derecha (Liau et al., 2004; Lehmann \& Clavien, 2010). 


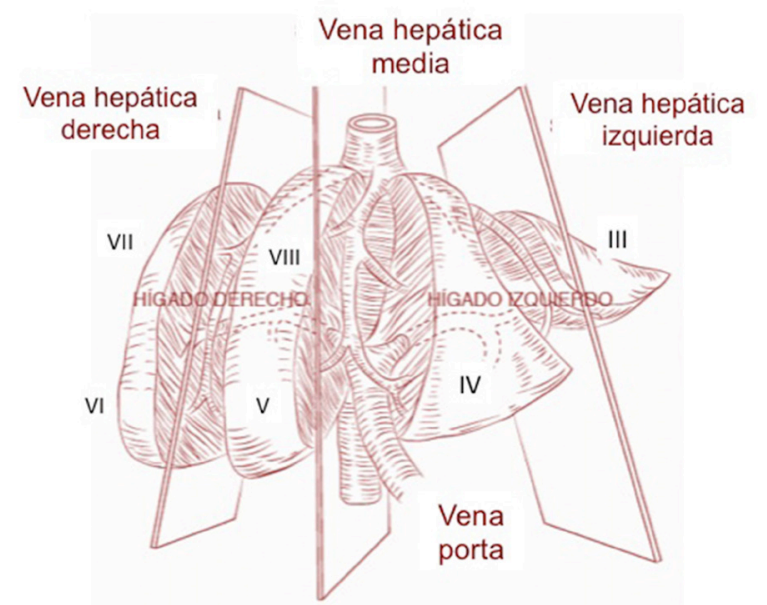

Fig. 2. Proyección de las venas hepáticas y planos de división del hígado en 4 secciones.

Lortat-Jacob, informó en 1952, de una resección anatómica hepática por cáncer. En 1953, Healey y Schroy sugirieron que el tamaño de los lóbulos derecho e izquierdo son similares (con base en el enfoque morfológico). Posteriormente, Couinaud en 1957, publicó una completa descripción de la anatomía segmentaria del hígado; lo que permitió a los cirujanos poder realizar cirugía hepática con base en una mayor comprensión de la anatomía vascular y biliar, permitiendo el tratamiento de enfermedades benignas y malignas con cifras de morbilidad y mortalidad cada vez menor; las que han ido mejorando de forma paulatina gracias al desarrollo tecnológico y del soporte quirúrgico perioperatorio (Lehmann \& Clavien; Azoulay et al., 2017; Calatayud et al., 2017).

En la década de los 90s, el acceso laparoscópico para la cirugía hepática comenzó a reportarse; destacando las publicaciones de Reich, Gagner, Azagra y Hüscher (Reich et al., 1991; Gagner et al., 1992; Azagra et al., 1996; Hüscher et al., 1997).

Otro hito importante ocurre en 2000, cuando el Comité Científico de la Asociación Internacional Hepatobilio-Pancreática (IHPBA), aprobó una nueva terminología, para poner fin a la confusión entre términos franceses y anglosajones, tanto anatómicos como quirúrgicos. Esta, se conoce como clasificación de Brisbane (Terminology Committee of the International Hepato-Pancreato-Biliary Association et al., 2000).

Una serie de otros hitos relevantes han ocurrido, los que se han desarrollado en base al conocimiento de la anatomía funcional y quirúrgica del hígado, y que a su vez, han agregado mayor conocimiento a ésta. Entre ellos, cabe destacar, el desarrollo del trasplante hepático, con Starzl y
Calne en la década de los 60s (Orozco-Zepeda, 2005); la ecografía intraoperatoria en 1976 con Makuuchi (Araki et al., 2014; Donadon et al., 2014; Prieto Martin, 2014), la hepatectomía ex-vivo (Pichlmayr et al., 1990), el ALPPS o "associating liver partition and portal ligation for staged hepatectomy" (Baumgart et al., 2011); etc.

\section{BASES ANATÓMICAS DE LAS RESECCIONES HE- PÁTICAS}

Los 8 segmentos anatómicos del hígado se delimitan por la distribución de los sistemas venoso hepático y portal (Figs. 3 y 4). Además, cada segmento tiene un flujo vascular y drenaje biliar independiente (Couinaud, 1999). Por consiguiente, es posible eliminar uno o más segmento (s) sin interrumpir el flujo sanguíneo ni biliar de los segmentos restantes (Abdel-Misih \& Bloomston).

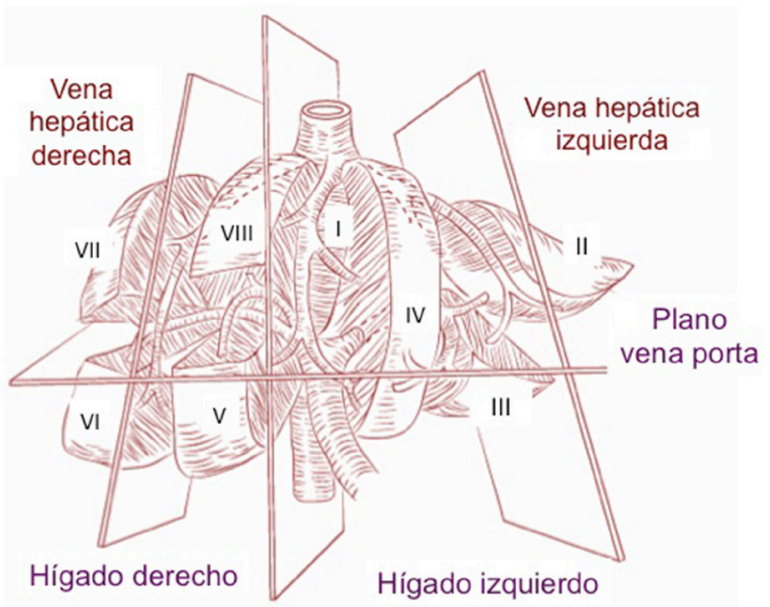

Fig. 3. Proyección de las venas hepáticas, bifurcación portal y división hepática en 8 segmentos.

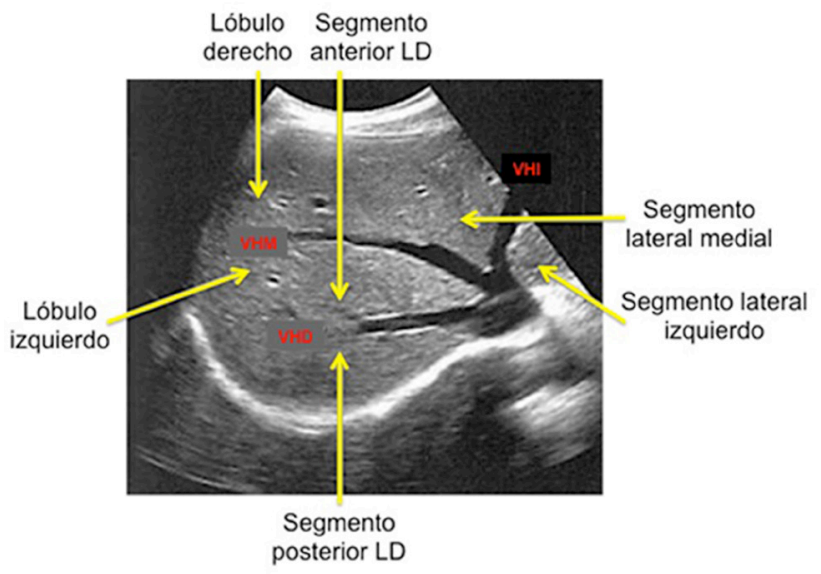

Fig. 4. Imagen ecotomográfica de la proyección de las venas hepáticas y su relación con los lóbulos y segmentos hepáticos. 
Basado en la propuesta de Brisbane, el hígado se puede dividir en 4 secciones: la sección anterior derecha (segmentos V y VIII), sección posterior derecha (segmentos VI y VII), sección medial izquierda (segmento IV) y sección lateral izquierda (segmentos II y III) (Terminology Committee of the International Hepato-Pancreato-Biliary Association et al.) (Tabla I) (Figs. 5 y $6)$.

Tabla I. Clasificación de la segmentación hepática según Brisbane, 2000.

\begin{tabular}{|c|c|c|c|}
\hline Término anatómico & $\begin{array}{c}\text { Segmentación } \\
\text { de } \\
\text { Couinaud } \\
\end{array}$ & Término quirúrgico & Esquema \\
\hline \multicolumn{4}{|c|}{$\begin{array}{c}\text { División de } \mathbf{1}^{\circ} \text { orden } \\
\text { dos separados por un plano medial, recorrido por la ven }\end{array}$} \\
\hline $\begin{array}{l}\text { Hígado derecho } \\
\text { o } \\
\text { Hemi-hígado derecho }\end{array}$ & $5-8( \pm 1)$ & $\begin{array}{l}\text { Hepatectomía derecha o } \\
\text { Hemi-hepatectomía derecha } \\
\text { (indicar } \pm \text { segmento } 1 \text { ) }\end{array}$ & 4 \\
\hline $\begin{array}{l}\text { Hígado izquierdo } \\
\text { o } \\
\text { Hemi-hígado izquierdo }\end{array}$ & $2-4( \pm 1)$ & $\begin{array}{l}\text { Hepatectomía izquierda o } \\
\text { Hemi-hepatectomía izquierda } \\
\text { (indicar } \pm \text { segmento } 1 \text { ) }\end{array}$ & 4 \\
\hline
\end{tabular}

División de $2^{\circ}$ orden

(planos interseccionales definidos por la vena hepática derecha y ligamentos redondo y falciforme)

Sección anterior derecha

Sección posterior derecha

Sección medial izquierda

4

Sección lateral izquierda
Seccionectomía medial izquierda, ó segmentectomía 4

Seccionectomía lateral izquierda, ó bisegmentectomía 2, 3

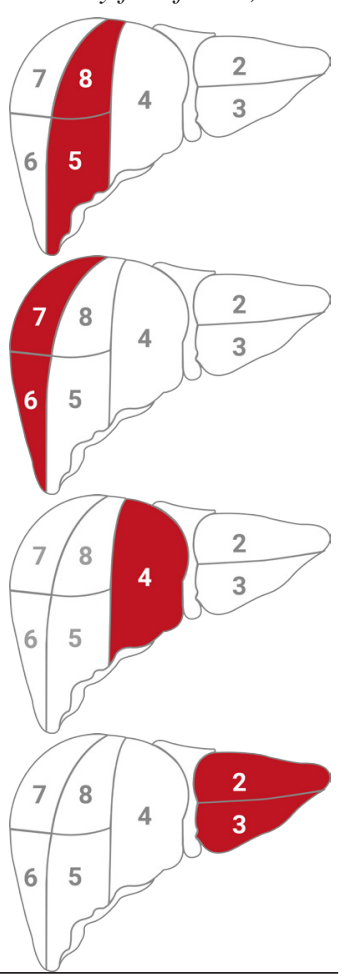


Tabla I. Clasificación de la segmentación hepáticade Brisbane, 2000 (2a parte)

\begin{tabular}{lccc}
\hline Término anatómico & $\begin{array}{c}\text { Segmentación } \\
\text { de } \\
\text { Couinaud }\end{array}$ & Término quirúrgico & Esquema \\
\hline
\end{tabular}

División de $2^{\circ}$ orden (según vena porta)

Sección anterior

derecho, ó paramediano

Sección posterior derecha, ó lateral derecho

Sección medial izquierda, ó paramediano izquierdo

Sección lateral izquierdo, ó posterior izquierdo

Seccionectomía anterior derecha, o paramediana derecha

Seccionectomía lateral medial, ó bisegmentectomía 3, 4

Seccionectomía lateral izquierda, ó posterior 2

Seccionectomía posterior derecha, ó lateral derecha paramediana izquierda, ó izquierda, ó segmentectomía

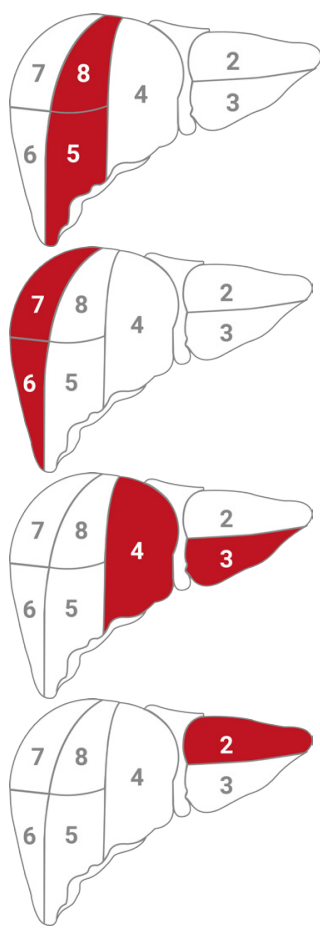

Otras divisiones de $2^{\circ}$ orden

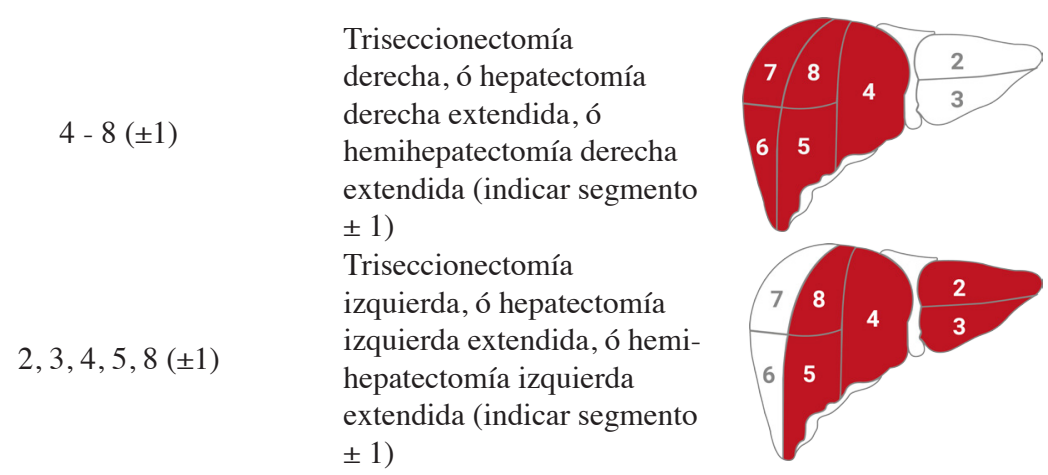

División de $3^{\circ}$ orden (según vena porta)

No tienen límites precisos, dependen de pedículos glissonianos)

Segmentos 1 al 9 continuos
Dos segmentos (1 al 9) en continuidad
Segmentectomía (por ej. segmentectomía 6)

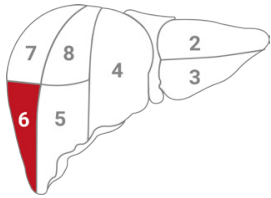

Bisegmentectomía (por ej. segmentectomía 5 y 6 )

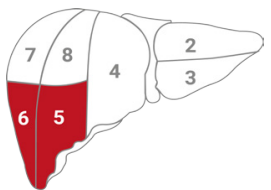



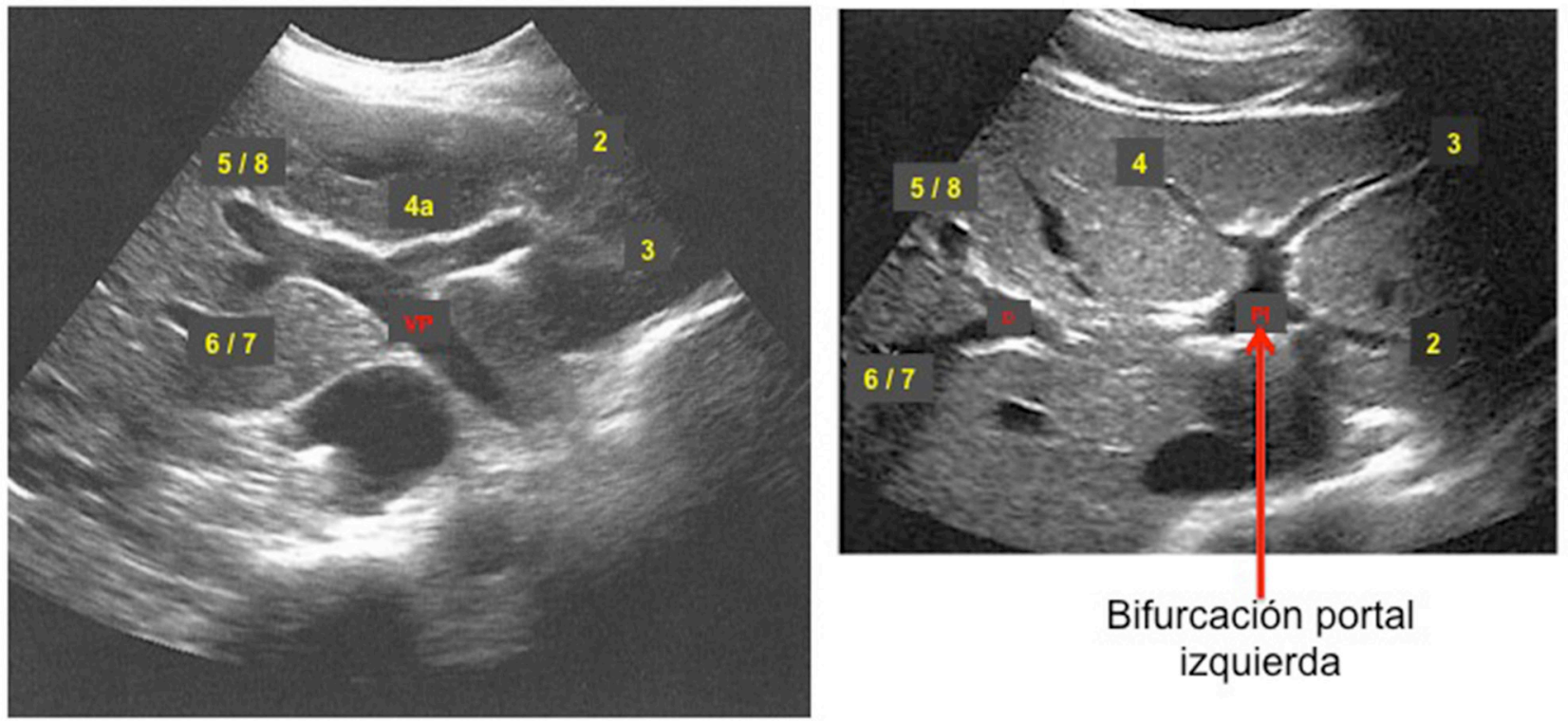

Fig. 5. Imágenes ecotomográficas de la bifurcación portal, distribución de las venas segmentarias y segmentos de ambos lóbulos.
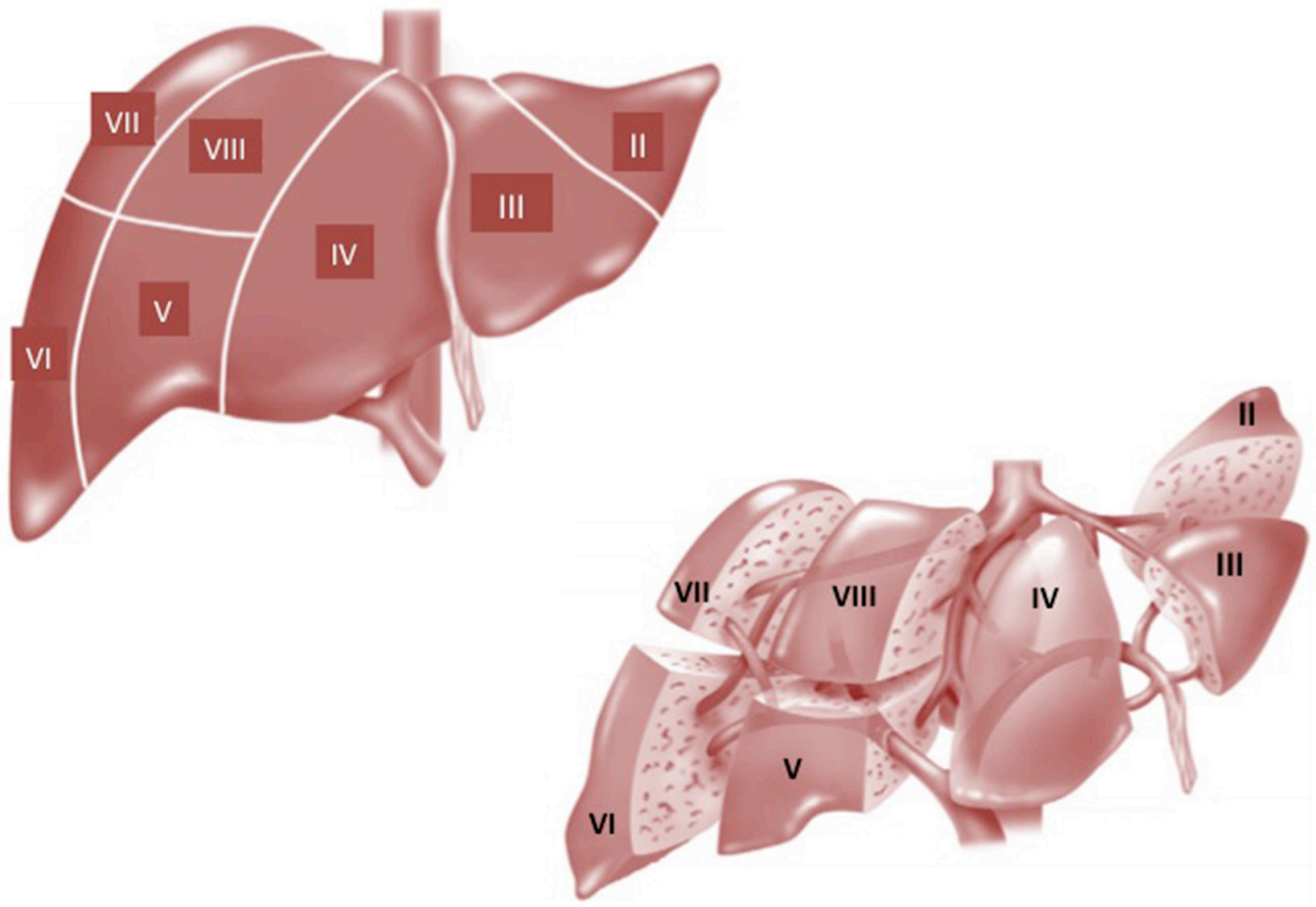

Fig. 6. Esquema de los 8 segmentos hepáticos. 
Por su parte, Terminologia Anatomica (FIPAT, 2011) divide al hígado a partir de la denominada "segmentación hepática portal: porciones, divisiones y segmentos [A05.8.01.033]":
A05.8.01.037 Porción hepática izquierda
A05.8.01.038 División lateral izquierda
A05.8.01.039 Segmento lateral izquierdo; Segmento II
A05.8.01.040 Segmento anterior lateral izquierdo; Segmento III
A05.8.01.041 División medial izquierda
A05.8.01.042 Segmento medial izquierdo; Segmento IV
A05.8.01.043 Porción posterior del hígado; Lóbulo caudado
A05.8.01.044 Segmento posterior; Lóbulo caudado; Segmento I
A05.8.01.045 Porción hepática derecha
A05.8.01.046 División medial derecha
A05.8.01.047 Segmento anterior medial derecho; Segmento V
A05.8.01.048 Segmento posterior medial derecho;Segmento VIII
A05.8.01.049 División lateral derecha
A05.8.01.050 Segmento anterior lateral derecho; Segmento VI
A05.8.01.051 Segmento posterior lateral derecho; Segmento VII

En relación al segmento II o segmento lateral izquierdo, se ha recomendado la inclusión, a dicho término, del adjetivo "posterior," quedando finalmente como segmento posterior lateral izquierdo (Carvallo et al., 2015). Esta propuesta de modificación terminológica se debe a que este segmento recibe una vena lateral izquierda, proveniente de la rama izquierda de la vena porta hepática, y que presenta un trayecto horizontal, posterior e izquierdo. Por lo tanto, debido a esto, y siguiendo lo establecido por el Programa Federativo Internacional de Terminología Anatómica, que se encarga de regular la comunicación en anatomía mediante modificaciones en la terminología, para lograr términos más claros y sobre todo con un valor preferentemente informativo y descriptivo, es que se sugiere esta incorporación del adjetivo mencionado y la modificación del término.
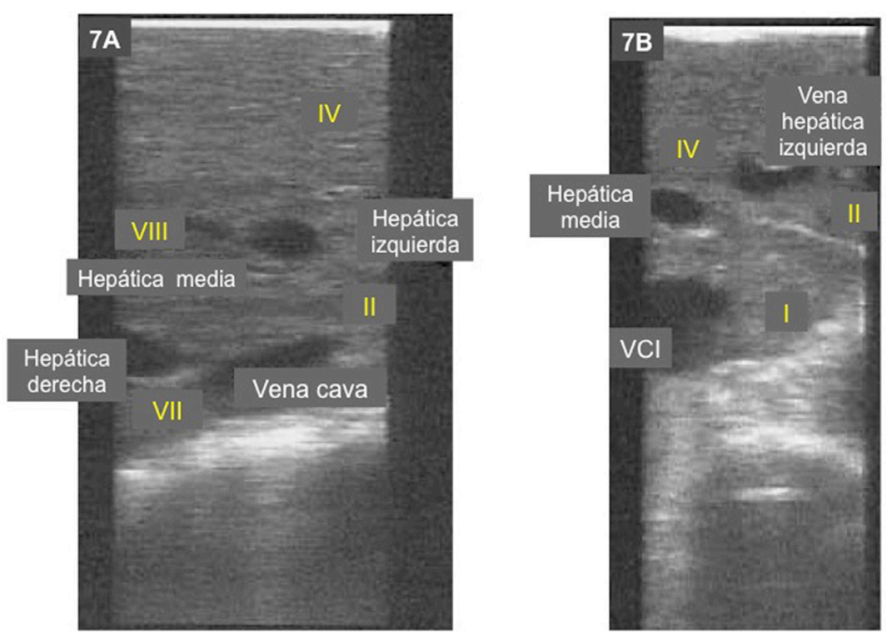

Fig. 7. Imágenes de ecografía intraoperatoria. VH derecha y media; y segmentos 2, 4, 8 y 7 (7A). VH media e izquierda; y segmentos 1,2 y 4 (7B).
Por otra parte, la cápsula fibrosa perivascular ("vaina de Glisson") rodea, en conjunto, a la arteria hepática propia, la vena porta y el conducto colédoco y continúa como capa fibrosa; de modo tal, que los pedículos seccionales derechos del hígado son accesibles quirúrgicamente en el hilio por medio de un acceso glissoniano intrahepático posterior (Launois et al., 1999). La ligadura del pedículo permite el control vascular, previo a la transección parenquimatosa. Por otra parte, el pinzamiento selectivo de un pedículo, permite demarcar él o los segmento (s) hepático (s) de interés y guía el plano de resección. Una alternativa a esta maniobra, es la técnica de cartografía por inyección de tinta (Makuuchi et al., 2002; Majno et al., 2014).

Los principales vasos de salida del hígado son las venas hepáticas derecha, media e izquierda; generalmente accesibles fuera del hígado, al que entran en la vena cava inferior (VCI). El control precoz de las venas hepáticas $(\mathrm{VH})$, permite minimizar la pérdida de sangre intraoperatoria. La presencia de una gran vena accesoria inferior al hígado derecho o una vena umbilical bien desarrollada al hígado izquierdo proporciona flexibilidad adicional en la realización de la resecciones segmentarias porque proporcionan drenaje venoso alternativo en el caso de que las venas hepáticas derecha o media, deban ser sacrificadas (Abdel-Misih \& Bloomston).

Utilidad de la ecografía intraoperatoria. La idea de realizar ecografía intraoperatoria (EIO), comenzó en 1960, cuando se utilizó para evaluar la existencia de coledocolitiasis; sin embargo, no fue hasta 1980 cuando se comenzó a utilizar con la tecnología que conocemos hoy (Kamtoh Tebug et al., 2012). Actualmente, es un elemento fundamental para realizar cirugía hepatobiliar, pues no sólo permite valorar la anatomía hepática (en especial, los elementos vasculares y biliares, y sus respectivas asociaciones), sino que también sirve como herramienta diagnóstica y terapéutica que permite evaluaciones precisa y eventuales tratamientos de lesiones focales (Donadon et al.).

Su uso nos permite preferentemente identificar las venas hepáticas (Figs. 7A y 7B), el pedículo portal y su distribución anatómica (Figs. 8A, 8B y 8C), las ramas izquierda y derecha de la vena porta hepática y sus respectivas ramas segmentarias; la vía biliar y su distribución; y aplicar eco-doppler (Schwarz et al., 2015).

Por ende, sus mayores usos son: Visualización de lesiones indetectables por palpación debido a su pequeño tamaño o a la existencia de cirrosis hepática 

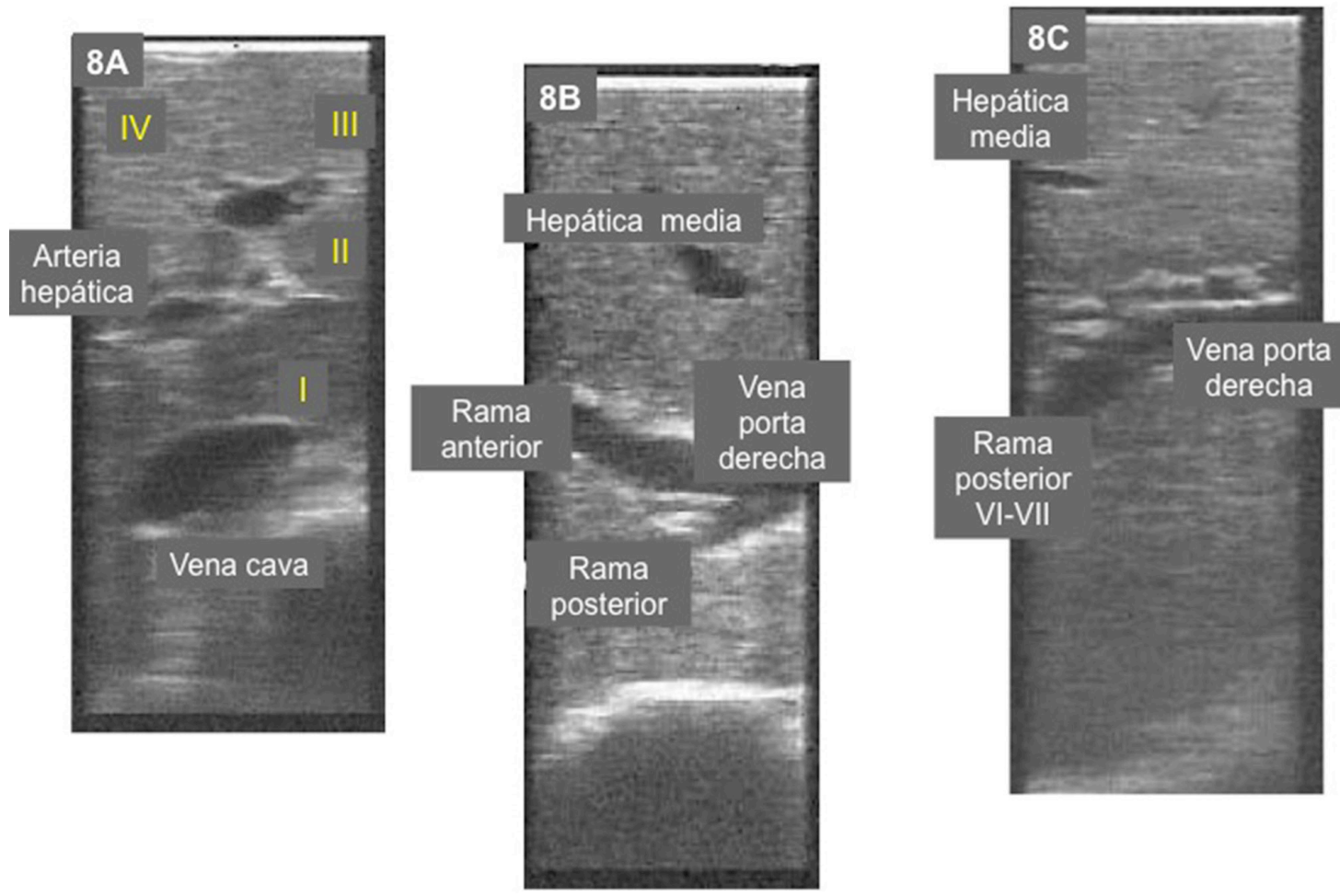

Fig. 8. Imágenes de ecografía intraoperatoria. VPizquierda y segmentos 1, 2, 3 y 4 (8A). VP derecha y rama anterior (segmentos 5 y 8) y posterior (segmentos 6 y 7); 8B. VP derecha y rama posterior (segmentos 6 y 7); 8C.

coexistente; identificación de las lesiones poco visibles o invisibles a la tomografía computarizada debido a la quimioterapia, pero que requieren resección; determinación de número y localización de lesiones; precisión de estado de avance de un tumor; determinación naturaleza y diferenciación de tumores benignos y malignos; visualización de émbolos neoplásicas en las venas; determinación del margen de resección; valoración del flujo vascular posterior a una resección; valoración del flujo sanguíneo de arterias, vena porta y venas hepáticas (agregando el doppler) (Lucchese et al., 2015; Schwarz et al.).

Justificación de la hepatectomía segmentaria. Existen varias razones para practicar resecciones segmentarias en vez de hepatectomías no anatómicas o atípicas. En primer término, una resección anatómica es oncológicamente superior, de lo que existe evidencia, por ejemplo, respecto de su efectividad en el tratamiento de las metástasis hepáticas de cáncer de colon y recto respecto de la resección en cuña; debido a que el porcentaje de margen positivo en resecciones no anatómicas es mayor $(16 \%$ a $30 \%)$ respecto de resecciones anatómicas (2\%); y mejor tasa de supervivencia en pacientes con resección anatómica (Scheele et al., 1990; DeMatteo et $a l ., 2000)$. Por otra parte, en casos de carcinoma hepatocelular, las resecciones segmentarias son más efectivas en el control de la satelitosis tumoral que se propaga por las ramas intrasegmentales (Scheele \& Stangl, 2000; Makuuchi et al., 2002). En segundo lugar, una hepatectomía segmentaria permite conservar parénquima respecto de una hepatectomía mayor; situación particularmente relevante en pacientes con cirrosis hepática. En tercer término, una resección segmentaria, permite reducir las pérdidas hemáticas, debido a que existe mejor control vascular del territorio a resecar respecto de una resección atípica, en la que existe mayor riesgo de desgarros venosos por falta de visión del plano de transección; y porque en la interfaz intersegmentaria existe menor vascularización (Majno et al.).

Elementos operacionales. Existe suficiente evidencia en relación con que la hemorragia intraoperatoria es el principal determinante del resultado perioperatorio de una hepatectomía (Neeff et al., 2009; Bennett et al., 2016); por ende, es fundamental utilizar estrategias tendientes a minimizar la pérdida hemática. Una de éstas, es mantener la presión venosa central por debajo de $5 \mathrm{mmHg}$ (minimizando la infusión de fluidos y restringiendo la transfusión intraoperatoria de sangre a menos que se pierda más del $25 \%$ del volumen sanguíneo (DeMatteo et al.), de modo tal de asegurar un flujo vascular de entrada y salida reducido (Melendez et al., 1998). 
Gestos quirúrgicos básicas en cirugía hepática. Se debe acceder al abdomen de forma adecuada, a través de una laparotomía amplia (en “J”, subcostal bilateral, Mercedes Benz) o de un acceso laparoscópico (dependiendo de la situación). Luego, se de be realizar una movilización completa del hígado, seccionando los ligamento redondo y suspensorio, los triangulares y coronales; luego de lo cual, se puede realizar con cierta comodidad una ecografía intraoperatoria (Fig. 9). Posteriormente, se recurre a la disección del pedículo hepático, con o sin maniobra de Pringle (Fig. 10), según el tipo de cirugía que se haya planificado (la colecistectomía puede ser necesaria o no). Luego, se ejecuta la transección del parénquima hepático, utilizando Kelly clasia, electrobisturí, haz de argón, Ligasure, CUSA, grapadoras vasculares, etc. (Lehmann \& Clavien), procediendo a la ligadura y sección de elementos vasculares y biliares que se encuentren en la línea de sección. La superficie cruenta se puede dejar tal cual, o se puede recurrir a un parche de omento. El uso de drenes es discutible, pero se pueden utilizar en casos de sospecha que pueda desarrollarse una filtración biliar. Se concluye con el cierre de la laparotomía.

A parte de las maniobras antes descritas, en una resección hepática se han de considerar algunas etapas fundamentales: a) las lesiones tumorales se deben localizar de forma precisa por medio de imágenes preoperatorias y por ecografía intraoperatoria (Makuuchi et al., 1985); b) movilizar el hígado seccionando sus elementos de sostén (ligamentos falciforme, triangulares, coronales (Fig. 11), hepatogástrico, separar la glándula suprarrenal derecha, seccionar y ligar las venas retro-

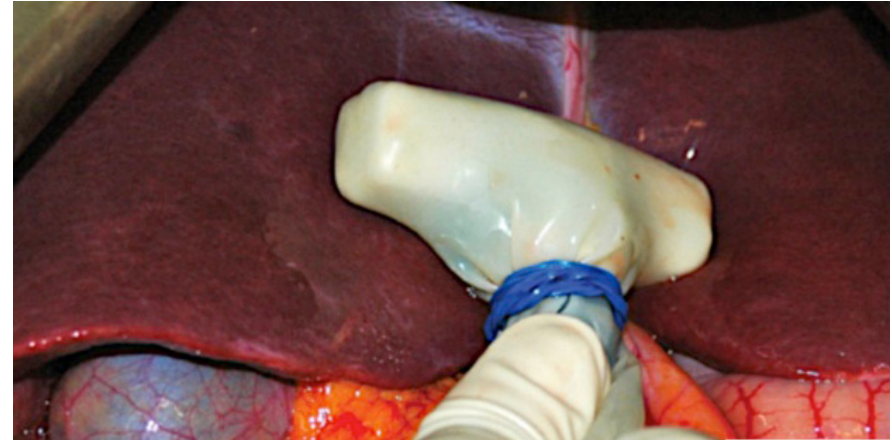

Fig. 9. Ecografía intraoperatoria. El transductor aplicado sobre la superficie hepática, desplazándose a través del ligamento suspensorio en dirección a las venas hepáticas.

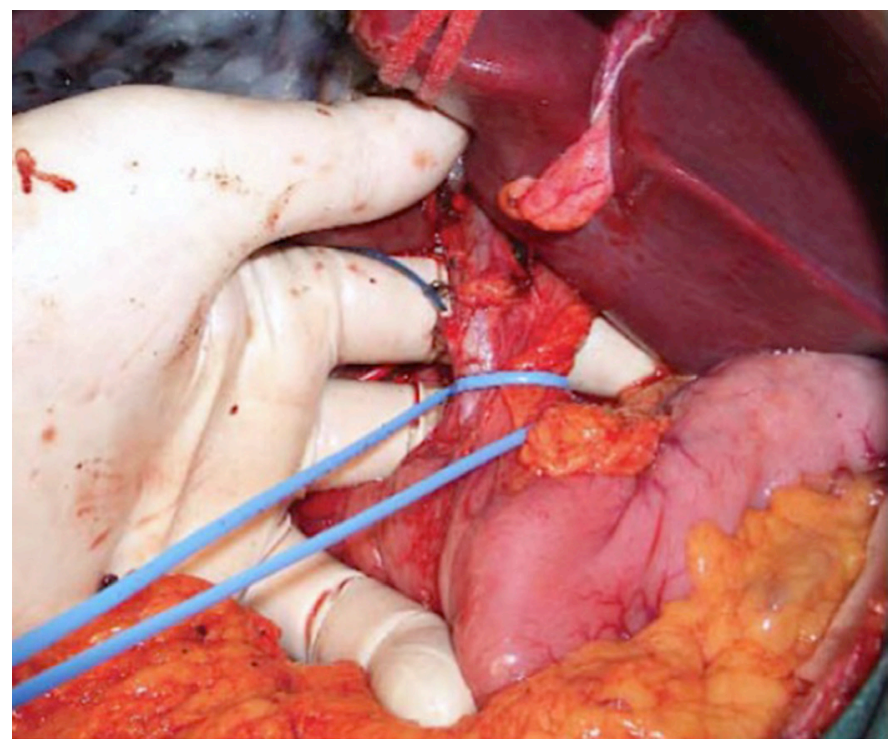

Fig. 10. Maniobra de Pringle. Se aprecia un vessel loop que rodea el pedículo hepático.

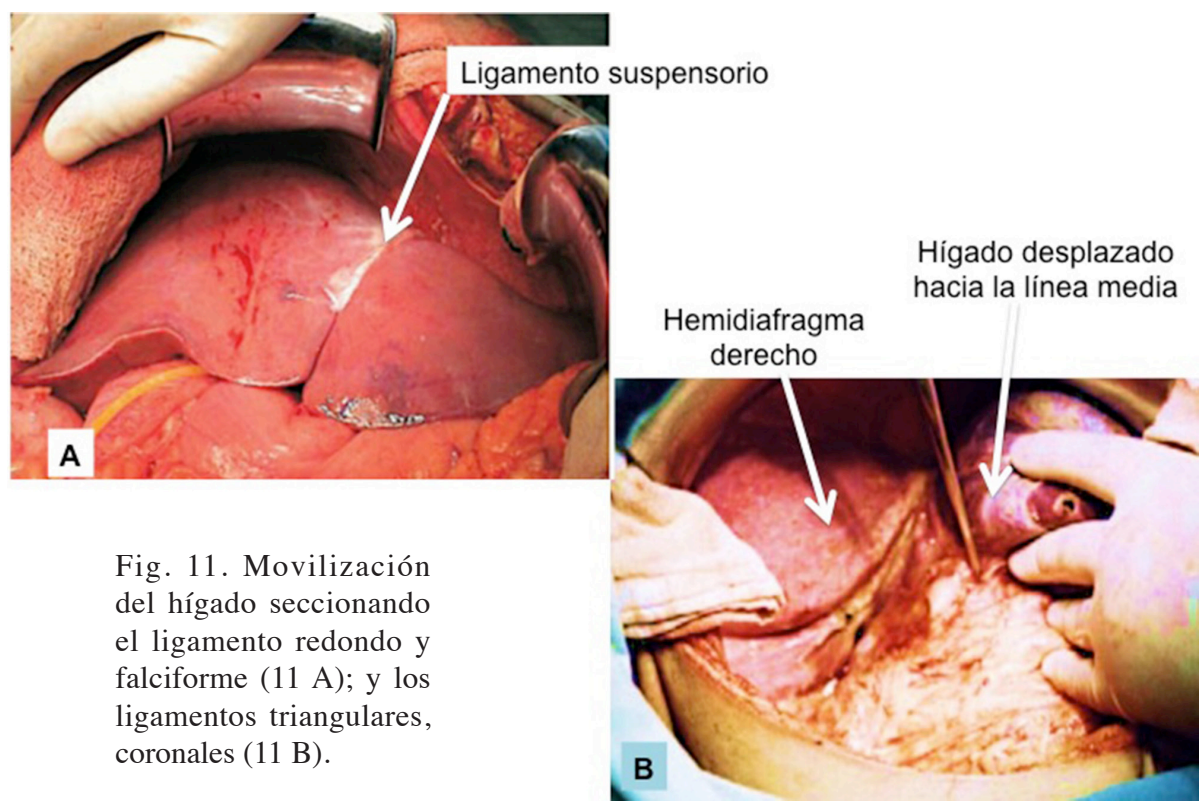


hepáticas o vénulas accesorias (Fig. 12); dependiendo de que tipo de hepatectomía se realizará); c) realizar un control vascular del hígado (pedículo hepático, venas hepáticas y VCI) (Fig. 13); y d) realizar la transección parenquimatosa (Fig. 14), seccionando y ligando vasos sanguíneos y conductos biliares interlobulillares. De este modo, las resecciones segmentarias pueden clasificarse en: segmentectomía, bisegmentectomía y segmentectomías múltiples (Celinski \& Gamblin, 2010; Fasel \& Schenk, 2013) (Tabla I ) (Fig. 15).

\section{Resecciones segmentarias. Segmentectomías}

Segmento I o Ióbulo caudado (segmentectomía I). Representa un gran desafío quirúrgico, debido a su localización y relación con la VCI y la vena porta hepática (Fig. 16). Se puede acceder desde la izquierda o la derecha realizando una hepatotomía. En casos de tumores de gran tamaño, es más seguro trabajar desde la derecha, ya que proporciona una mejor exposición para el flujo de entrada y salida (Fan et al., 2001). Sin embargo, el acceso desde la izquierda tras la movilización completa del lóbulo izquierdo también es válida; para ello, se debe seccionar el omento menor y el ligamento dorsal que se encuentra posterior a la VCI (el flujo principal proviene de la rama izquierda de la vena porta hepática y de la arteria hepática izquierda, que son accesibles en la base de la fisura umbilical). Por último, se puede considerar la opción de dividir el hígado a lo largo del plano principal para acceder al lóbulo caudado en la cara, que expone la vena hepática media (Yamamoto et al., 2014). Se ha

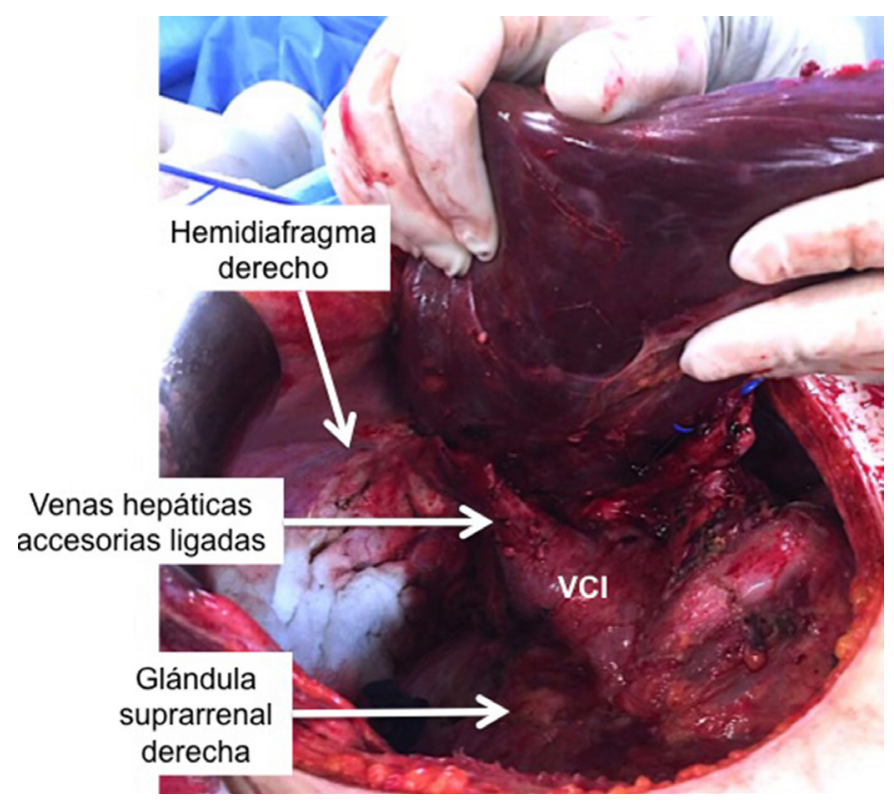

Fig. 12. Movilización completa del hígado separando la glándula suprarrenal derecha, seccionando y ligando las venas hepáticas accesorias. Se aprecia la VCI disecada.

de considerar que las venas retrohepáticas o vénulas accesorias que drenan directamente a la VCI constituyen la mayor parte del flujo de salida, son frágiles y deben ser ligadas. Por otra parte, también se debe tomar en cuenta la vena hepática media, que puede lesionarse durante la transección. En ocasiones, puede ser útil controlar las venas hepáticas izquierda y media precozmente, para visualizar la vena hepática media directamente usando la

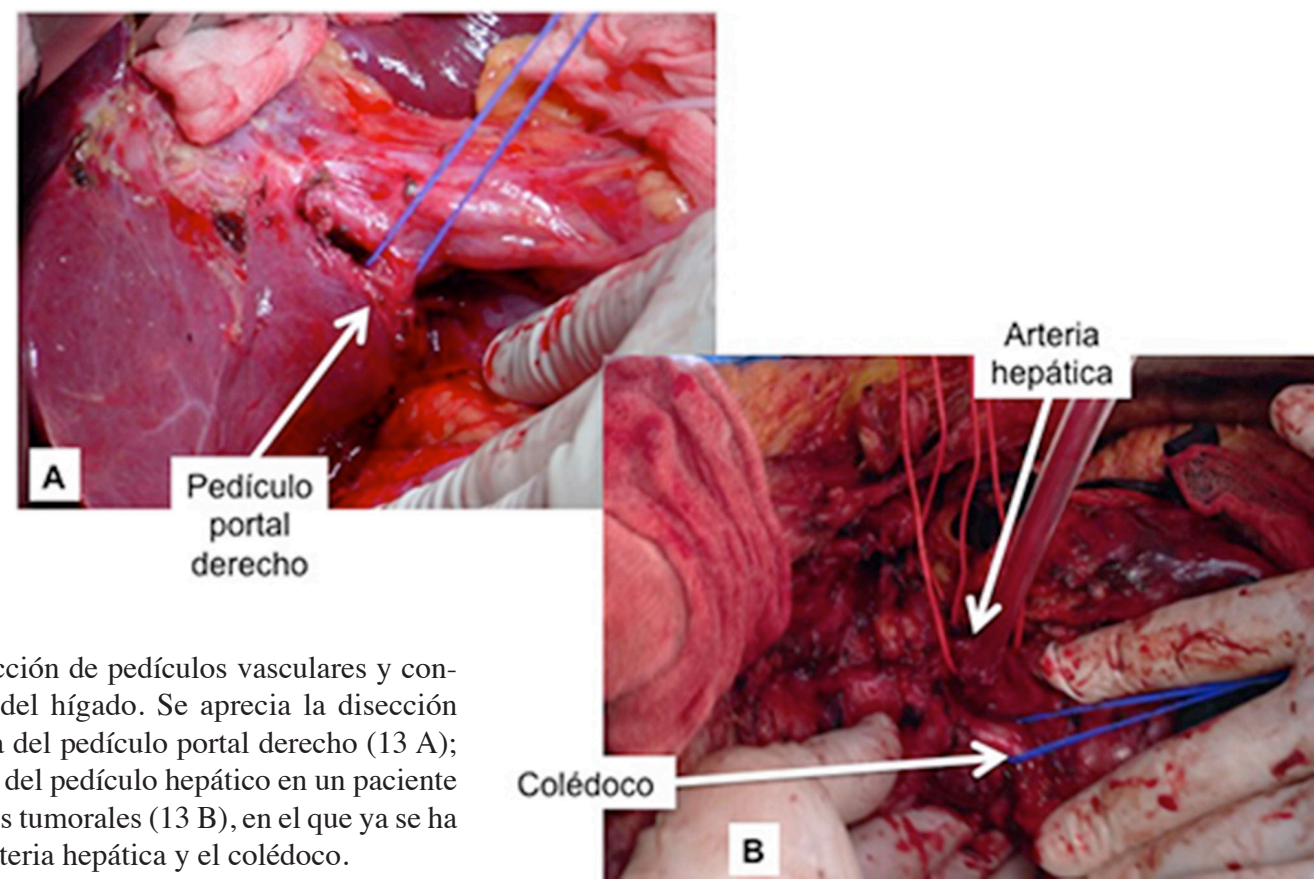



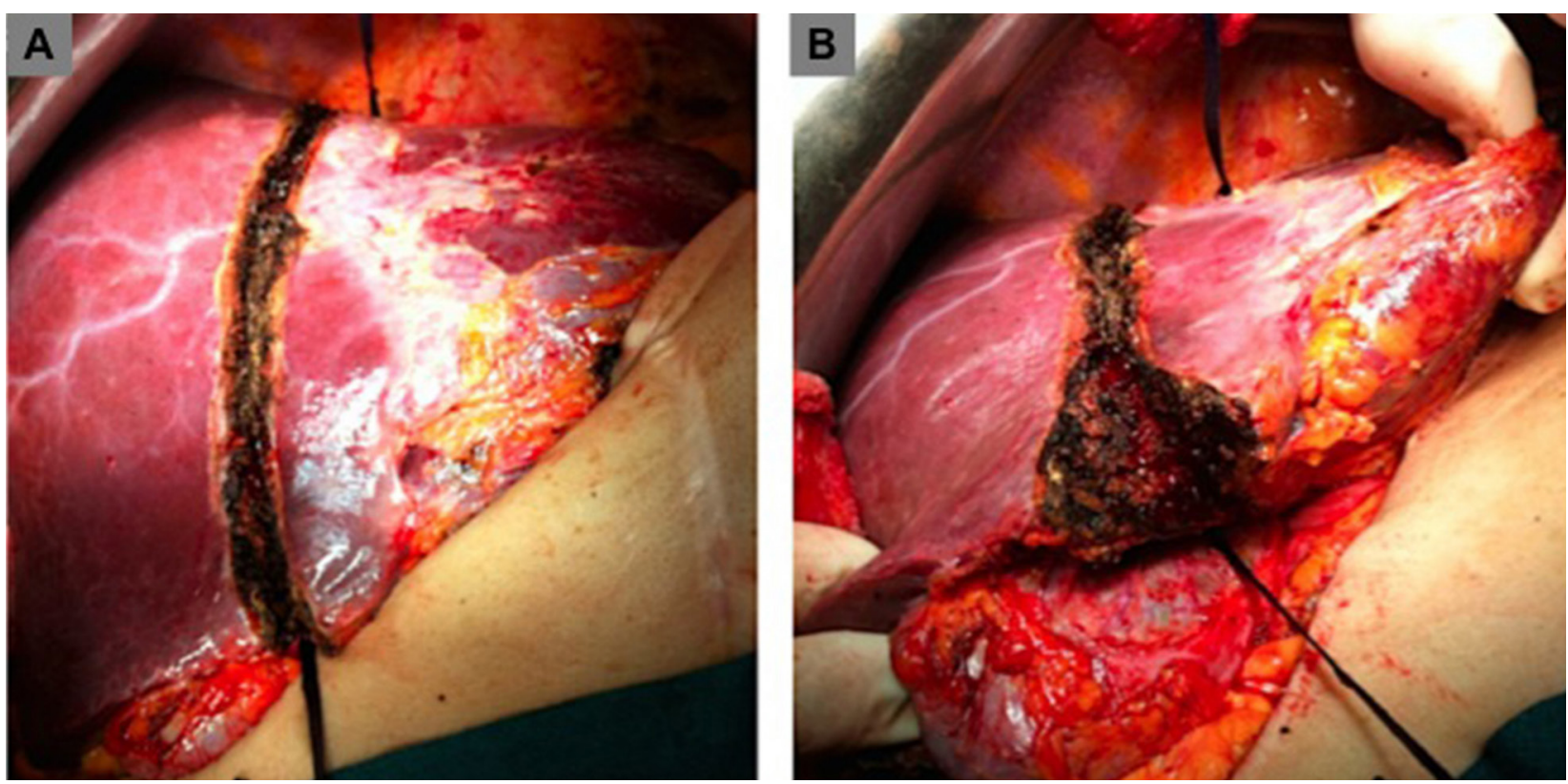

Fig. 14. Transección suspendida del parénquima hepático en una bisegmentectomía izquierda. Junto con la sección parenquimatosa, se van seccionando y ligando vasos sanguíneos y conductos biliares interlobulillares.
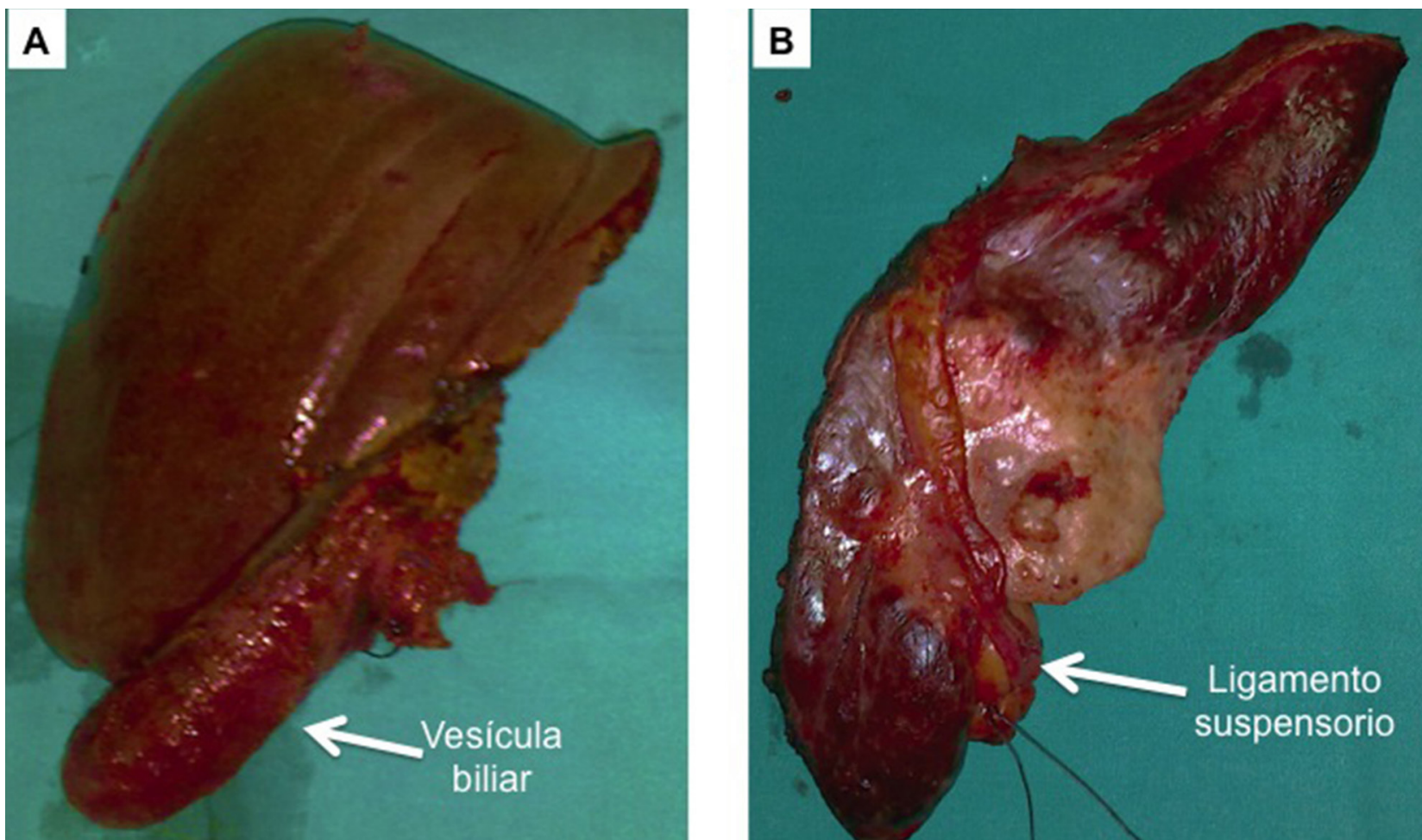

Fig. 15. Piezas quirúrgicas de hepatectomía derecha o hemi-hepatectomía derecha producto de un colangiocarcinoma (15 A); y de una hepatectomía izquierda, o hemi-hepatectomía izquierda, producto de un hepatocarcinoma en un paciente con cirrosis hepática (15 B).

técnica del hígado dividido. Una vez que el caudado está completamente movilizado, el puente parenquimatoso que une el caudado con los segmentos VI y VII se puede observar de forma clara para proceder a su transección (Fasel \& Schenk; Majno et al.).
Segmentos II o III (segmentectomías II y III). Es poco habitual realizar una segmentectomía aislada de los segmentos II o del III; lo que se explica por la mayor simpleza de realizar una bisegmentectomía II-III. Sin embargo, en pacientes con cirrosis hepática y escasa reserva funcional del hígado, 

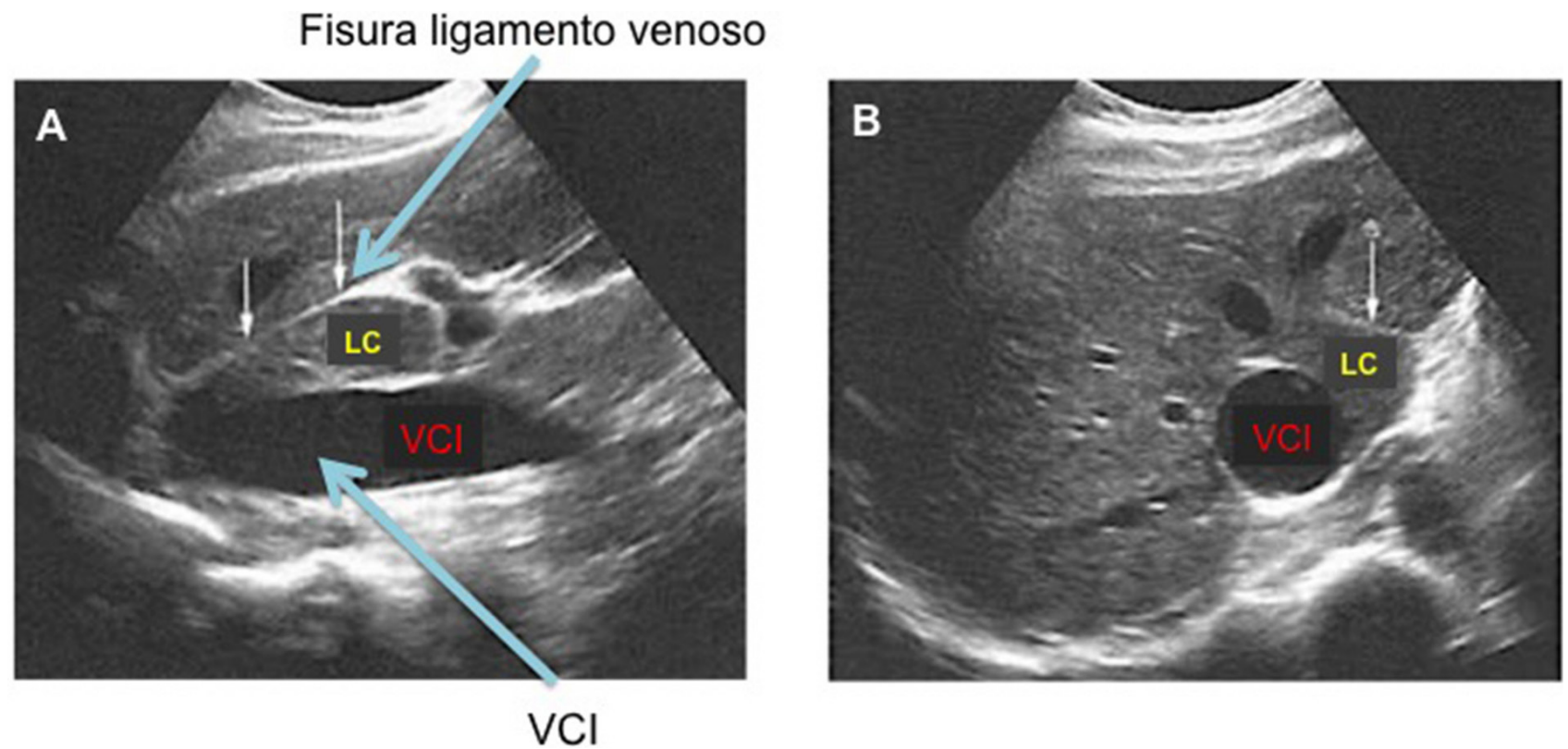

Fig. 16. Lóbulo caudado y su relación con la VCI en una visión sagital (16 A); y transversal (16 B).

o cuando se requiere practicar resecciones segmentarias múltiples, la resección aislada puede ser necesaria. Ya sea para la exéresis del II como del III, se liga el pedículo correspondiente, conservando la vena hepática izquierda porque proporciona el único drenaje venoso al segmento restante. Los pedículos de los segmentos II y III salen directamente de la porción umbilical de la rama izquierda de la vena porta hepática; por lo que para aislarlos, se debe levantar el lóbulo izquierdo utilizando tracción en el ligamento falciforme dividido. A continuación, se secciona el puente parenquimatoso entre los segmentos III y IV. Luego, se diseca la fisura umbilical a la izquierda de la vena porta y se procede a ligar el pedículo correspondiente, lo que delimitará el límite de sección. Se finaliza el procedimiento con la transección parenquimatosa en un plano antero-craneal oblicuo intentando preservar la vena hepática izquierda (Celinski \& Gamblin).

Segmento IV (segmentectomías IV). Es un procedimiento complejo de realizar, debido entre otras, a que este segmento se subdivide en dos subsegmentos, IVA y IVB; con base en los pedículos vasculares. Por ello, es que se puede extirpar en bloque la totalidad del segmento IV, o una de sus porciones de forma aislada. En primer término, se debe disecar el hilio hepático para liberar las estructuras portales de la base del segmento IV. Luego, se debe realizar una disección intrahepática de la porción umbilical del pedículo portal izquierdo (en especial, en casos de tumores cercanos a la fisura umbilical). De lo contrario, el el plano de transección puede ir a la derecha del ligamento falciforme para evitar lesiones en la porción umbilical de la rama izquierda de la vena porta hepática. Una vez ligado los pedículos del seg1536 mento IV, se delimitará el límite de sección. El control del flujo de salida no se obtiene hasta después de la transección parenquimatosa, la que comienza en el margen inferior del hígado, traccionando el espécimen con dos tractores de hilo (primero por la izquierda, luego por la derecha, para finalizar con una sección transversal del límite superior para separarla del segmento I posterior). Dependiendo de la localización del tumor, puede ser necesario sacrificar la vena hepática media.

Segmento V y VI (bisegmentectomía VI y VII o seccionectomía posterior derecha). Esta, es una de las más frecuentes de realizar. Se utiliza la incisura de Gans, surco de Rouvière, o incisura hepatis dextra como hito anatómico-quirúrgico para localizar el pedículo seccional posterior; pues esta presente en aproximadamente un $70 \%$ de los pacientes (Reynaud et al., 1991; Dahmane et al., 2013). El drenaje venoso de esta sección es predominantemente por la vena hepática derecha, aunque se estima que en un $25 \%$ de los casos puede ocurrir a través de una vena accesoria, directamente a la VCI (Makuuchi et al., 1985). Al pinzar este pedículo, se logra demarcar el área de sección. A continuación se realiza la transección, finalizando con la ligadura y sección de la vena hepática derecha (especialmente en casos de cercanía de un tumor a esta estructura anatómica (Celinski \& Gamblin).

Segmento VII (segmentectomía VII). Es poco común de realizar; sin embargo, en un hígado cirrótico, puede ser una valiosa opción para eliminar un pequeño tumor hepático. La dificultad técnica de una segmentectomía VII es su pedículo vascular orientado hacia atrás y localizado bajo el hemidiafragma derecho; lo que hace que su disección y 
aislamiento sea técnicamente compleja, requiriéndose de una movilización hepática completa hacia la izquierda. La transección parenquimatosa, debe seguir el plano transversal a través del hilio, y a medida que se va profundizando, se logra identificar el pedículo vascular del segmento VII. Una vez asegurado, se continua con la transección hacia la vena hepática derecha, la que suele ser necesario sacrificar. Existe una vena hepática accesoria derecha en el 25\% de los individuos (Makuuchi et al., 1985). En ausencia de esta vena, puede ser más prudente proceder con la seccionectomía posterior derecha.

Segmento VIII (segmentectomía VIII). Representa un verdadero desafío técnico, debido al riesgo de lesionar las venas hepáticas derecha y media (ambas limitan este segmento). Se puede acceder a través de una hepatotomía con base en la fisura portal principal conectando la fosa de la vesícula biliar y el margen izquierdo de la VCI. La otra opción es pinzando el pedículo de la sección anterior derecha o el pedículo hepático derecho. La ventaja de ésta última es que al hacerlo se demarcan los límites de la resección derecha e izquierda del segmento VIII (lo que puede ser dificultoso). Por otra parte, se ha de considerar que el límite inferior del segmento VIII corresponde al plano transversal a través del hilio hepático; por ende, durante la división del límite inferior, se debe buscar tanto el pedículo de la sección anterior derecha como el pedículo del segmento VIII (que una vez identificado se debe ligar); por ello es fundamental un campo quirúrgico exangüe para evitar lesionar o ligar erróneamente el pedículo del segmento V. Una vez demarcada el área de sección, se procede a la transección parenquimatosa, intentando preservar la vena hepática derecha. La exéresis termina desprendiendo el espécimen de la superficie anterior de VCI entre los troncos principales de las venas hepáticas media y derecha (Celinski \& Gamblin).

\section{Bisegmentectomías o seccionectomías}

Seccionectomía posterior derecha (segmentos VI y VII). La exéresis de cualquiera de estos segmentos, es relativamente simple debido a que su localización es accesible y la distancia de las estructuras vasculares principales es razonable. Primero se debe proceder al pinzamiento selectivo a nivel hiliar, de los pedículos de la sección anterior o posterior derecha, lo que permitirá marcar el segmento a resecar. A continuación, se ha de acceder a los pedículos segmentarios correspondientes, seccionarlos y ligarlos; teniendo en consideración la vasculatura de los segmentos VIII y VII. Si se ha de realizar una segmentectomía del V, se sugiere efectuar primero la colecistectomía; posterior a lo cual, continuar con la transección, la que debe seguir una línea ventral a la incisura de Gans para evitar lesiones en el pedículo de la sección posterior derecha (Fasel \& Schenk).
Seccionectomía anterior derecha (segmentos V y VIII). Es una hepatectomía muy poco frecuente (indicado especialmente en hígados cirróticos con tumores localizados en el sector anterior derecho con invasión del límite entre los segmentos V y VIII). Para su ejecución, es imprescindible movilizar ampliamente el hígado, asegurando un control vascular. Se sugiere comenzar con la ligadura del pedículo seccional anterior derecho para un mejor control vascular. Posteriormente, demarcar el área de sección en la vaina de Glisson con electrobisturí. La transección parenquimatosa, se realiza a lo largo de la fisura portal principal, hacia la vena hepática derecha. El dilema en estos casos es la decisión de sacrificar la vena hepática media (lo que afecta el drenaje venoso del segmento IV, que tendrá que realizarse por colaterales de la vena umbilical), lo que dependerá de la proximidad del tumor a la vena (Celinski \& Gamblin).

Seccionectomía lateral izquierda (segmentos II y III). Es una de las hepatectomías más frecuentes de realizar. El enfoque quirúrgico es el mismo que para efectuar una segmentectomía del II o del III. Se sugiere acceder al principal de la vena hepática izquierda girando el hígado hacia la derecha después de la movilización completa del lóbulo izquierdo. Luego, se sugiere identificar el túnel que existe detrás de la vena hepática izquierda y delante de la VCI; debido a que éste ángulo es mejor expuesto dividiendo la porción cefálica del ligamento ducto venoso o ligamentum venosum (a la izquierda del lóbulo caudado). A continuación se puede instalar una cinta vascular detrás de la vena hepática izquierda, lo que permite mejor control vascular (se puede también aislar de este modo tanto la vena hepática izquierda como la media). Se encuentra indicada en casos de lesiones que comprometan ambos segmentos (Fasel \& Schenk).

\section{Segmentectomía múltiples}

Resección hepática central (segmentos IV, V y VIII). También se denomina hepatectomía central y mesohepatectomía. Es una técnica poco utilizada. En el fondo consiste en una combinación de la técnica de resección de segmento IV y sección anterior derecha. Para ello, se debe realizar una disección del hilio y la fisura umbilical, para tener un adecuado control vascular. Posteriormente, se debe aislar los pedículos de la sección anterior derecha y del segmento IV. La transección parenquimatosa puede comenzar a la derecha de la fisura umbilical (o dentro de ella si el tumor estuviese muy cerca), continuando hasta el tronco principal de la vena hepática media, evitando ligar la rama umbilical del pedículo portal izquierdo. Por otra parte, se debe ligar el pedículo seccional anterior derecho, lo que determinará la demarcación del límite de sección, para comenzar la transección. Esta, se debe continuar por el plano de la vena hepática derecha hasta encontrar el plano de sección izquierdo (se 
debe proceder con cautela, ya que los segmentos a resecar quedarán prácticamente colgando, por lo que una tracción desmedida puede provocar una lesión de la vena hepática media, con la consecuente hemorragia). Una vez aquí, se debe dividir la base de la vena hepática media; y se finaliza con un parche de omento para cubrir la superficie cruenta del hígado. Con esta hepatectomía, se elimina la vesícula biliar, los segmentos IV, V y VIII; y la vena hepática media en bloque, dejando intacto el lóbulo caudado (Celinski \& Gamblin; Fasel \& Schenk).

MANTEROLA, C.; DEL SOL, M.; OTTONE, N.\& OTZEN, T. Radiological and surgical anatomy of the liver and fundamentals of the various options liver resections. Int. J. Morphol., 35(4):15171524, 2017.

SUMMARY: The liver is a solid organ which is most relevant for physiology. It is a potential site for cystic and solid (primary and secondary) benign and malignant tumor lesions. Therefore, thorough knowledge of its radiological and surgical anatomy is important. Historical background of liver resections began with Berta in 1716, who was the first to carry out the procedure. In 1888, Lagenbuch performed the first programmed liver resection and subsequently, in 1889 Keen performed the very first left hepatic lobectomy, followed by Webde in 1910, who performed the first right hepatic lobectomy. Later in 1957, Couinaud recorded a complete description of the segmental anatomy of the liver, providing a greater surgical understanding of the hepatic morphology, for approach in various pathologies. A fundamental milestone in the development of the liver study was the establishment of the "Brisbane Classification" by the Scientific Committee of the International Hepatobiliary-Pancreatic Association, which ended previous confusion between the French and Anglo-Saxon terminology. Furthermore, within the scope of anatomy, the introduction of Terminología Anatómica, by the International Federative Program of Anatomical Terminology (FIPAT) which depends on the International Federation of Associations of Anatomists ( IFAA), established the anatomical terms for the liver The objective of this manuscript is to provide a schematic summary of the surgical and radiological anatomy of the liver, on which the different options for liver resections are based.

KEY WORDS: Hepatic anatomy; Liver surgery; Hepatic resections; Hepatectomies; Sectioning.

\section{REFERENCIAS BIBLIOGRÁFICAS}

Abdel-Misih, S.R. \& Bloomston, M. Liver anatomy. Surg.Clin. North Am., 90(4):643-53, 2010.

Araki, K.; Conrad, C.; Ogiso, S.; Kuwano, H. \& Gayet, B. Intraoperative ultrasonography of laparoscopic hepatectomy: key technique for safe liver transection. J. Am. Coll. Surg., 218(2):e37-41, 2014.

Azagra, J. S.; Goergen, M.; Gilbart, E. \& Jacobs, D. Laparoscopic anatomical (hepatic) left lateral segmentectomy-technical aspects. Surg. Endosc., 10(7):758-61, 1996.
Azoulay, D.; Bhangui, P.; Pascal, G.; Salloum, C.; Andreani, P.; Ichai, P.; Saliba, F. \& Lim, C. The impact of expanded indications on short-term outcomes for resection of malignant tumours of the liver over a 30 year period. HPB (Oxford), 19(7):638-48, 2017.

Baumgart, J.; Lang, S. \& Lang, H. A new method for induction of liver hypertrophy prior to right trisectionectomy: A report of three cases. HPB (Oxford), 13(Suppl. 2):71-2, 2011.

Bennett, S.; Baker, L.; Shorr, R.; Martel, G. \& Fergusson, D. The impact of perioperative red blood cell transfusions in patients undergoing liver resection: a systematic review protocol. Syst. Rev., 5:38, 2016.

Bismuth, H. Revisiting liver anatomy and terminology of hepatectomies. Ann. Surg., 257(3):383-6, 2013.

Calatayud, D.; Sánchez Cabús, S.; Sampson, J.; Resendiz, A.; Molina, V.; Fondevila C.; Fuster, J. \& García-Valdecasas, J. C. Hepatic resection: a safe and effective surgery. Cir. Esp., 95(8):437-46, 2017.

Carvallo, E.; Carvallo, P.; Coello, R. \& Vásquez, B. Segmento II del Hígado ¿Segmento Lateral Izquierdo o Segmento Posterior Lateral Izquierdo? Int. J. Morphol., 33(2):433-5, 2015.

Celinski, S. A. \& Gamblin, T. C. Hepatic resection nomenclature and techniques. Surg. Clin. North Am., 90(4):737-48, 2010.

Couinaud, C. Liver anatomy: portal (and suprahepatic) or biliary segmentation. Dig. Surg., 16(6):459-67, 1999.

Dahmane, R.; Morjane, A.; Starc, A. Anatomy and surgical relevance of Rouviere's sulcus. Scientific World Journal 2013:254287, 2013.

DeMatteo, R. P.; Palese, C.; Jarnagin, W. R.; Sun, R. L.; Blumgart, L. H. \& Fong, Y. Anatomic segmental hepatic resection is superior to wedge resection as an oncologic operation for colorectal liver metastases. $J$. Gastrointest. Surg., 4(2):178-84, 2000.

Del Cura, J. L.; Pedraza, S.; Gayete, A. Radiología Esencial. Madrid, Médica Panamericana, 2010.

Donadon, M.; Costa, G. \& Torzilli, G. State of the art of intraoperative ultrasound in liver surgery: current use for staging and resection guidance. Ultraschall Med., 35(6):500-11, 2014.

Fan, J.; Wu, Z. Q.; Tang, Z. Y.; Zhou, J.; Qiu, S. J.; Ma, Z. C.; Zhou, X. D. $\&$ Yu, Y. Q. Complete resection of the caudate lobe of the liver with tumor: technique and experience. Hepatogastroenterology, 48(39):808$11,2001$.

Fasel, J. H. \& Schenk, A. Concepts for liver segment classification: Neither old ones nor new ones, but a comprehensive one. J. Clin. Imaging Sci., 3:48, 2013.

Federative International Programme on Anatomical Terminologies. Terminologia Anatomica. 2nd. ed. Thieme Verlag, Stuttgart, 2011.

Gagner, M.; Rheault, M. \& Dubuc, J. Laparoscopic partial hepatectomy for liver tumor. Surg. Endosc., 6:99, 1992.

Hüscher, C. G.; Lirici, M. M.; Chiodini, S. \& Recher, A. Current position of advanced laparoscopic surgery of the liver. J.R. Coll. Surg. Edinb., 42(4):219-25, 1997

Juza, R. M. \& Pauli, E. M. Clinical and surgical anatomy of the liver: a review for clinicians. Clin. Anat., 27(5):764-9, 2014.

Kamtoh Tebug, G.; Kubisz, A.; Ko?odziejczyk, P.; Pach, R.; Sierz e ga, M. \& Kulig, J. Intraoperative ultrasonography in hepatobiliary surgery. Pol. Przegl. Chir., 84(12):657-67, 2012.

Launois, B.; Maddern, G. \& Tay, K. H. The Glissonian approach of the hilum. Swiss Surg., 5(3):143-6, 1999.

Lehmann, K. \& Clavien, P. A. History of hepatic surgery. Surg. Clin. North Am., 90(4):655-64, 2010.

Liau, K. H.; Blumgart, L. H. \& DeMatteo, R. P. Segment-oriented approach to liver resection. Surg. Clin. North Am., 84(2):543-61, 2004.

Lucchese, A. M.; Kalil, A. N.; Schwengber,A.; Suwa, E. \& Rolim de Moura, G. G. Usefulness of intraoperative ultrasonography in liver resections due to colon cancer metastasis. Int. J. Surg., 20:140-4, 2015.

Majno, P.; Mentha, G.; Toso, C.; Morel, P.; Peitgen, H. O. \& Fasel, J. H. Anatomy of the liver: an outline with three levels of complexity--a further step towards tailored territorial liver resections. J. Hepatol., 60(3):654-62, 2014.

Makuuchi, M.; Imamura, H.; Sugawara, Y. \& Takayama, T. Progress in 
surgical treatment of hepatocellular carcinoma. Oncology, $62 \mathrm{Suppl}$. $1: 74-81,2002$.

Makuuchi, M.; Hasegawa, H. \& Yamazaki, S. Ultrasonically guided subsegmentectomy. Surg. Gynecol. Obstet., 161(4):346-50, 1985.

Melendez, J. A.; Arslan, V.; Fischer, M. E.; Wuest, D.; Jarnagin, W. R.; Fong, Y. \& Blumgart, L. H. Perioperative outcomes of major hepatic resections under low central venous pressure anesthesia: blood loss, blood transfusion, and the risk of postoperative renal dysfunction. $J$. Am. Coll. Surg., 187(6):620-5, 1998.

Neeff, H.; Makowiec, F.; Harder, J.; Gumpp, V.; Klock, A.; Thimme, R.; Drognitz, O. \& Hopt, U. T. Hepatic resection for hepatocellular carcinoma--results and analysis of the current literatura. Zentralbl. Chir., 134(2):127-35, 2009.

Orozco-Zepeda, H. A little history about the liver transplantation. Rev. Investig. Clin., 57(2):124-8, 2005.

Pichlmayr, R.; Grosse, H.; Hauss, J.; Gubernatis, G.; Lamesch, P. \& Bretschneider, H. J. Technique and preliminary results of extracorporeal liver surgery (bench procedure) and of surgery on the in situ perfused liver. Br. J. Surg., 77(1):21-6, 1990.

Prieto Martin, M. Ecografía intraoperatoria en la cirugía hepática. Radiología., 56:1517, 2014.

Reich, H.; McGlynn, F.; DeCaprio, J. \& Budin, R. Laparoscopic excision of benign liver lesions. Obstet. Gynecol., 78(5 Pt. 2):956-8, 1991.

Reynaud, B. H.; Coucoravas, G. O. \& Giuly, J. A. Basis to improve several hepatectomy techniques involving the surgical anatomy of incisura dextra of Gans. Surg. Gynecol. Obstet.,172(6):490-2, 1991.

Scheele, J.; Stangl, R. \& Altendorf-Hofmann, A. Hepatic metastases from colorectal carcinoma: impact of surgical resection on the natural history. Br. J. Surg., 77(11):1241-6, 1990.

Scheele, J. \& Stangl, R. Segment-Orientated Anatomical Liver Resections. In: Blumgart, L. H. \& Fong, Y. (Eds.). Surgery of the Liver, Biliary Tract, and Pancreas. Philadelphia, Saunders Elsevier, 2000. pp.1743-64.

Schwarz, L.; Vibert, E. \& Sa Cunha, A. Intra-operative ultrasonography of the liver. J. Visc. Surg., 152(4):245-50, 2015.

Skandalakis, J. E.; Skandalakis, L. J.; Skandalakis, P. N. \& Mirilas, P. Hepatic surgical anatomy. Surg. Clin. North Am., 84(2):413-35, 2004.

Terminology Committee of the International Hepato-Pancreato-Biliary Association; Strasberg, S. M.; Belghiti, J.; Clavien, P. -A.; Gadzijev, E.; Garden, J. O.; Lau, W. -Y.; Makuuchi, M. \& Strong, R. W. The Brisbane 2000 Terminology of Liver Anatomy and Resections. HPB (Oxford), 2(3):333-9, 2000.

Yamamoto, M.; Katagiri, S.; Ariizumi, S.; Kotera, Y.; Takahashi, Y. \& Egawa, H. Tips for anatomical hepatectomy for hepatocellular carcinoma by the Glissonean pedicle approach (with videos). J. Hepatobiliary Pancreat. Sci., 21(8):E53-6, 2014.

\author{
Dirección para correspondencia: \\ Dr. Carlos Manterola, MD, PhD. \\ Departamento de Cirugía y CEMyQ \\ Universidad de La Frontera \\ Temuco \\ CHILE
}

E-mail: carlos.manterola@ufrontera.cl

Recibido : 05-08-2017

Aceptado: 29-09-2017 